\title{
Frontières
}

\section{Les momies victoriennes et leur postérité}

Enquête sur la fonction spectaculaire et symbolique du cadavre momifié

\section{Fanny Robles}

Volume 23, numéro 2, printemps 2011

Enquêtes sur le cadavre : 2. Fantastique

URI : https://id.erudit.org/iderudit/1007585ar

DOI : https://doi.org/10.7202/1007585ar

Aller au sommaire du numéro

Éditeur(s)

Université du Québec à Montréal

ISSN

1916-0976 (numérique)

Découvrir la revue

Citer cet article

Robles, F. (2011). Les momies victoriennes et leur postérité : enquête sur la fonction spectaculaire et symbolique du cadavre momifié. Frontières, 23(2), 21-25. https://doi.org/10.7202/1007585ar

\section{Résumé de l'article}

Défi de l'homme lancé à la mort, le cadavre momifié soulève un certain nombre de questions psychanalytiques. Son potentiel spectaculaire a fait la fortune des momies égyptiennes dans l'Angleterre victorienne. Objet érotique et macabre, la momie est aussi un fétiche impérial, et apparaît dans la littérature puis sur les écrans comme une figure de la vengeance et du mal. 
Résumé

Défi de l'homme lancé à la mort, le cadavre momifié soulève un certain nombre de questions psychanalytiques. Son potentiel spectaculaire a fait la fortune des momies égyptiennes dans I'Angleterre victorienne. Objet érotique et macabre, la momie est aussi un fétiche impérial, et apparaît dans la littérature puis sur les écrans comme une figure de la vengeance et du mal.

Mots clés: mort-momie-féticheempire-gothique-Égypte.

\begin{abstract}
The mummified corpse stands as a challenge to death, and raises a few psychoanalytical questions. Its aptitude for the show has made Egyptian mummies very popular in Victorian England. On top of being an erotic and macabre object, the mummy is also an Imperial fetish and enters literature and, later, Hollywood's movie screens, as a revengeful and evil character.
\end{abstract}

Keywords: death-mummy-fetishempire-gothic-Egypt.

\section{Les momies victoriennes}

\section{et leur postérité} Enquête sur la fonction spectaculaire
et symbolique du cadavre momifié

\author{
Fanny Robles, M.A., \\ allocataire-moniteure, \\ Université Toulouse II-Le Mirail (France).
}

«La chair changera de nature, le corps prendra un autre nom; même celui de cadavre [...] ne lui demeurera pas longtemps; il deviendra un je ne sais quoi, qui n'a point de nom dans aucune langue.» Ces mots, que Bossuet emprunte à Tertullien pour son oraison funèbre du Père Bourgoing (Bossuet, 1659, p. 59), rappellent la fatalité à laquelle l'homme se sait soumis par sa condition mortelle. Le retour à la poussière originelle semble constituer l'horizon de l'existence humaine, preuve de son inscription dans le cycle naturel. Mais c'est sans compter sur la propension de l'humanité à maîtriser la nature et à faire fi des données biologiques en utilisant la technique. La momification devient ainsi la réponse de choix à la désagrégation du corps. Si elle existe à l'état de processus naturel, les tissus se conservant par dessèchement, que ce soit dans les sables ou dans les glaces, la transformation d'un cadavre en momie nous intéresse ici en tant qu'intervention humaine sur le corps du défunt, à travers l'embaumement.

Les premières occurrences de ce phénomène remontent à l'Égypte ancienne, où la momification des morts constitue l'étape indispensable d'un rite religieux. Aussi étrange que cela puisse paraître, c'est à travers des pratiques cannibales que l'Occident prend conscience de ces momies au Moyen Âge, puisqu'elles sont alors prisées pour leurs vertus médicinales ${ }^{1}$. C'est au XVI ${ }^{\text {e }}$ siècle que la momie égyptienne devient objet de collection pour les Européens, et que commence la fascination macabre dont le XIX ${ }^{\mathrm{e}}$ siècle constitue l'apogée ${ }^{2}$. C'est cette dernière époque que notre étude se propose d'explorer, en s'intéressant en particulier à la GrandeBretagne, où les momies égyptiennes connaissent une fortune exceptionnelle.

Nous étudierons la dimension spectaculaire du corps momifié à l'époque victorienne, en nous penchant dans un premier temps sur les enjeux de la momification pour l'individu. En examinant l'exemple particulier de la société britannique au XIX ${ }^{\mathrm{e}}$ siècle, nous tenterons de comprendre pourquoi la fascination pour les momies y est plus forte qu'ailleurs en Europe. Dans un deuxième temps, nous étudierons la momie victorienne à l'aune de son contexte géopolitique, en nous y intéressant comme objet de musée, mais aussi figure centrale du roman d'aventures impérial, avant d'aborder la survivance hollywoodienne du mythe.

\section{BANDELETTES, PSYCHANALYSE ET SPECTACLE: LES ENJEUX DE LA MOMIFICATION}

Désir d'éternité momifiée, mais aussi désir morbide pour l'éternelle momie, la fascination pour ce corps imperméable au temps s'inscrit dans un contexte particulier, où l'existence sociale passe souvent par le théâtre, la mort ne faisant pas exception.

\section{RÉPONSE FÉTICHISTE À LA MORT}

Avant de nous plonger dans l'époque victorienne, il n'est pas sans intérêt de 
revenir sur la pratique de la momification et de s'interroger sur sa portée psychologique. Pour comprendre sa signification dans le rapport de l'homme à la mort, on peut étudier les soins thanatiques, qui participent à ce que Louis-Vincent Thomas appelle la «retenue du mort» (Thomas, 1985, p. 141). D'origine très ancienne, ce qui fut jadis la toilette du défunt a aujourd'hui été remplacé dans les sociétés occidentales par un véritable traitement chimique qui permet de préserver l'intégrité physique du cadavre durant plusieurs jours, voire plusieurs mois. Étape nécessaire du processus de deuil, cette résistance provisoire à la putréfaction des chairs est tout aussi symbolique que réelle et possède une dimension spectaculaire nécessaire, comme le souligne Thomas : «Lavé et paré, le mort offre une image magnifiée de sa personne comme pour donner un démenti solennel au pouvoir dissolvant de la mort » (Thomas, 1985, p. 149).

Cette préservation momentanée du corps dans son apparence de vie permet la domination de ce que Sigmund Freud désigne comme le principe de plaisir ${ }^{3}$ sur le principe de réalité ${ }^{4}$ : "L'acharnement à prolonger l'illusion de la vie est une conduite instinctive pour repousser l'échéance de la coupure»(Thomas, 1985, p. 147). L'habitude de parler au mort sur son lit mortuaire, présente dans de nombreuses cultures, fait partie intégrante de ce processus. Si l'on passe maintenant au cas de la momification, qui suppose une «retenue du mort» non plus temporaire mais éternelle, la relation au cadavre change de nature et est susceptible de prendre un caractère pathologique. La relation au corps du défunt peut ainsi devenir fétichiste, selon un mécanisme de remplacement. Dans son étude de la momie comme fétiche, Kelly Hurley rappelle l'interprétation freudienne qui définit le fétiche comme un objet qui vient compenser pour l'enfant l'absence de phallus maternel. Cela peut sembler quelque peu éloigné de ce qui nous préoccupe ici, mais Hurley retient de Freud l'idée que le fétichisme procède à la substitution d'un objet fantasmatique mais puissant, à un objet perdu ou susceptible de l'être (Hurley, 2008, p. 189): dans le cas qui nous préoccupe il s'agit de la substitution du cadavre momifié à la personne vivante disparue.

Indépendamment de tout contexte historique, cette déviance potentielle, inhérente à la persistance du cadavre dans son intégrité, offre un matériel de choix à la fiction, en particulier dans le registre de l'horreur. C'est le cas de la relation que Norman Bates entretient avec sa mère dans Psycho d'Alfred Hitchcock. Norman passe son temps libre à empailler des animaux, la taxidermie étant ici un équivalent animalier de la momification. On remarquera qu'il empaille exclusivement des oiseaux et qu'Horus, l'un des plus anciens dieux égyptiens, associé à la résurrection de son père Osiris, est traditionnellement représenté avec une tête de faucon. Bates dit vivre avec sa mère invalide, et cette dernière semble être l'auteure de crimes atroces dont il couvre les traces. Ce n'est qu'à la fin du film que l'on découvre l'effroyable vérité: Norman a empoisonné et embaumé sa mère, dont il emprunte la personnalité. Il reproduit sa voix, porte ses vêtements, et tue en son nom. C'est le décalage entre le réalisme du corps momifié de la mère, dont les orbites vides semblent fixer le spectateur, et la folie de Norman qui crée l'horreur. Selon le psychiatre qui l'examine à la fin du film: "he was simply doing everything possible to keep the illusion of his mother being alive» (Hitchcock, 1960, 101'). Nous sommes ici face à une perversion de la "retenue du mort» nécessaire au deuil. Même si Psycho est une fiction datant de 1960, la fascination morbide pour le corps momifié exploité par Hitchcock fait déjà trembler au XIX ${ }^{\mathrm{e}}$ siècle.

\section{INDÉPENDAMMENT DE TOUT}

CONTEXTE HISTORIQUE,

\section{CETTE DÉVIANCE POTENTIELLE,}

INHÉRENTE À LA PERSISTANCE

DU CADAVRE DANS SON

INTÉGRITÉ, OFFRE UN MATÉRIEL

DE CHOIX À LA FICTION,

\section{EN PARTICULIER DANS \\ LE REGISTRE DE L'HORREUR.}

PLACE DE LA MOMIE DANS L'APPROCHE VICTORIENNE DE LA MORT

Si l'on s'intéresse maintenant à l'époque victorienne, deux adjectifs pourraient qualifier la perception de la mort alors prédominante: horrible et théâtrale. Catharine Arnold souligne l'état particulièrement déplorable des cimetières des villes industrielles britanniques au début du XIX ${ }^{\mathrm{e}}$ siècle, quand la surpopulation de ces lieux de recueillement les transforme en lieux d'horreur. Les cercueils sont bien souvent défoncés pour palier le manque d'espace et les corps, entassés dans des fosses. Cette insalubrité à proximité des logements entraîne des épidémies de choléra. À cela s'ajoute la présence des Resurrection Men qui, avant l'Anatomy Act de $1832^{5}$, utilisent les cimetières comme des réserves de cadavres frais avec lesquels ils fournissent les facultés de médecine (Arnold, 2006, p. 94-122). On ne peut s'étonner alors du goût prononcé de la littérature anglaise pour les morts-vivants comme Frankenstein ou Dracula, étant donné la réalité morbide qui entoure alors les vivants.

La solution est alors de construire des cimetières à l'extérieur des grandes villes sur de vastes étendues, à l'image du Highgate Cemetery qui apparaît au nord de Londres en 1839. Mais une place dans les cimetières périphériques n'est pas à la portée de toutes les bourses, et les funérailles restent un moment privilégié de discrimination sociale. L'art funéraire se fait le reflet dans la mort de la richesse amassée par le défunt de son vivant et, à partir des années 1820, l'arrivée d'antiquités égyptiennes en Grande-Bretagne lance une vague d'égyptomanie sur la classe moyenne et l'aristocratie britanniques, les tombes s'ornant de monuments de style égyptien. Il est intéressant de remarquer que la crémation des morts, qui ne devient légale en Grande-Bretagne qu'en 1884 , se développe pour deux raisons: le progrès hygiénique qui contraste avec l'insalubrité des cimetières, et l'économie qu'elle représente en comparaison du faste inutile des enterrements traditionnels. Or, la crémation constitue l'archétype de la dissolution du corps, à l'opposé de la volonté de préservation extrême de l'embaumement.

À cette présence horrible de la mort, sublimée par le travail d'ornementation ostentatoire des caveaux, s'ajoute un certain fantasme du retour à la vie, entretenu par les spectacles scientifiques en vogue au $\mathrm{XIX}^{\mathrm{e}}$ siècle. Dès la fin du XVIII ${ }^{\mathrm{e}}$ siècle, on donne des expériences publiques portant sur le "galvanisme» ou l'étude des liens entre l'électricité et le système nerveux: des membres amputés semblent reprendre vie sous l'effet du courant. Ainsi, les premiers romans de momies mettent en scène la résurrection du corps momifié par l'électricité, à l'image de The Mummy! A Tale of the Twenty-Second Century... de Jane Webb (1827), et « Some Words with a Mummy» d'Edgar Alan Poe (1845). Dans la réalité, des spectacles d'un autre type viennent rendre vie aux momies: les séances de débandelettages. Le voyage en Égypte se fait de plus en plus courant, et les aristocrates qui peuvent se le permettre ramènent une momie de leurs péripéties orientales. La morale étant préservée derrière un alibi scientifique, les débandelettages ont également lieu dans 
les sociétés savantes; les sociétaires invitent leurs proches et amis et se livrent à un spectacle aux accents érotiques et nécrophiles, à rattacher à un certain romantisme noir, qui répond selon Renan Pollès à un double exotisme: celui de l'Orient et celui de la mort (Pollès, 2001, p. 112-119) ${ }^{6}$.

\section{CRÉATURE DE FREAK SHOW}

Si les momies égyptiennes sont exhibées dans un contexte de recherche scientifique, devant un public de classe sociale relativement aisée, la momification de leurs contemporains intéresse également les Victoriens, sur les scènes plus populaires des freak shows. Le succès de ce type de spectacle, qui consiste à exhiber des êtres humains jugés anormaux, n'est pleinement compréhensible que si l'on prend en compte l'importance cruciale des notions de limite et de classification pour l'époque victorienne. Dans Imperial Leather : Race, Gender, and Sexuality in the Colonial Contest, Anne McClintock souligne l'importance de démarcations rigides (rigid boundaries) dans la société britannique au XIX $\mathrm{X}^{\mathrm{e}}$ siècle, et l'existence de contre-modèles moraux, nécessaires à la définition identitaire de la bourgeoisie. Des figures de la transgression apparaissent alors : la prostituée (qui, à l'opposé de la respectable "Angel in the House», transgresse la limite entre la sphère domestique et la sphère privée), l'ouvrier, associé au danger socialiste, qui menace les limites entre classes sociales et perturbe l'ordre public, le terroriste irlandais qui met en danger l'intégrité du Royaume, et enfin l'indigène, certes lointain, qui questionne l'intégrité de l'Empire et soulève le problème des différences raciales (McClintock, 1995, p. 21-74).

Les freaks incarnent la transgression, dans leur statut souvent hybride. À une époque où la science est plus que jamais passionnée par les classifications, que ce soit en botanique, en zoologie ou en anthropologie (avec la classification des races humaines), les hommes et femmes qui semblent échapper aux catégories, de façon plus ou moins exagérée - car on sait la propension de ces spectacles à la supercherie -, excitent l'intérêt des foules. Un spécimen nous semble particulièrement intéressant: il s'agit de Julia Pastrana, une indigène mexicaine qui souffre d'hypertrichose (une pilosité développée lui recouvre la totalité du corps) et d'hyperplasie gingivale (ses gencives sont si développées qu'elles paraissent constituer une seconde rangée de dents) (Stern, 2008, p. 201). Généralement vêtue d'une robe à la mode, elle fait démonstration de sa très belle voix et des trois langues qu'elle maîtrise. Son apparence hors norme la positionne au

VÉRITABLE FÉTICHE IMPÉRIAL, LA MOMIE JOUE UN RÔLE PARTICULIER

DANS L'IMAGINAIRE COLONIAL VICTORIEN, CE QUI LAISSE DES TRACES

JUSQUE DANS LE CINÉMA HOLLYWOODIEN.

croisement de nombreuses catégories, à la fois homme et femme, humaine et animale, sauvage et civilisée (Stern, 2008, p. 206).

À l'âge de vingt-six ans, Pastrana meurt en couches, ainsi que son enfant. Et lorsque Theodore Lent, époux et imprésario de l'exhibée, décide de faire embaumer sa femme et leur fils, puis de les exposer derrière une cage de verre, il ajoute une autre transgression à l'actif de la défunte: celle de la limite entre la vie et la mort. Jadis exhibée en tant que "Baboon Lady» ou «Bear Woman», elle est désormais connue sous le nom de «Embalmed Nondescript». Selon Janet Browne et Sharon Messenger, le fait que l'exhibée soit morte donne au spectateur la possibilité de l'observer sans gêne (Stern, 2008, p. 214; Browne et al., 2003, p. 159). La conservation de Julia Pastrana en momie éternelle permet de fixer une forme d'essence de la déviance, d'incarner pleinement les angoisses victoriennes concernant l'altérité raciale, sexuelle, et finalement l'altérité absolue que constitue la mort.

\section{LA MOMIE : FÉTICHE IMPÉRIAL}

Ce lien entre angoisses psychanalytiques et spectacle du corps momifié nous amène à envisager la momie victorienne comme ce que Renan Pollès appelle le «retour du colonisé» ou "l'irruption au cœur du monde civilisé du fantôme de l'Orient pour se venger» (Pollès, 2001, p. 152). Véritable fétiche impérial, la momie joue un rôle particulier dans l'imaginaire colonial victorien, ce qui laisse des traces jusque dans le cinéma hollywoodien.

\section{OBJECTIFICATION CULTURELLE}

La momie est avant tout un corps devenu objet, et sa valeur marchande, dans le contexte géopolitique victorien, est grandement attachée à l'identité culturelle du corps conservé sous les bandelettes. L'instabilité de la gouvernance égyptienne dans les premières décennies du $\mathrm{XIX}^{\mathrm{e}}$ siècle, permet à l'Angleterre et à la France, par l'entremise de leurs consuls, de «découvrir» un certain nombre de monuments funéraires enfouis dans les sables et de les vider de leurs richesses et de leurs morts, ces derniers constituant sans doute une richesse autrement plus grande, sur le plan symbolique. Selon Pollès, l'expédition d'Égypte lancée par Napoléon en 1798 «innova un nouveau type de conquête coloniale où le contrôle des peuples passe par celui de leur histoire et fera souvent de la quête archéologique un préalable à la conquête militaire et du pillage des antiquités un préalable au pillage des richesses naturelles » (Pollès, 2001, p. 92). Institution emblématique du $\mathrm{XIX}^{\mathrm{e}}$ siècle, le musée devient un instrument de choix dans la domination impériale, puisque la gloire culturelle du pays conquis s'y trouve conservée, étiquetée, possédée par le pays conquérant. Ella Shohat et Robert Stam parlent à ce propos de «museological fetishizing of cultures » (Shohat et al., 1994, p. 153).

L'égyptologie naît avant tout comme une science impériale, instrument politique d'un Occident qui « aspire à l'universalisme et s'instaure comme l'héritier de toutes les grandes civilisations et le gardien de leurs vestiges, qu'ils fussent littéraires ou matériels, les indigènes dégénérés étant jugés indignes de cet héritage » (Pollès, 2001, p. 92). Seul l'archéologue européen ou américain est apte à déterminer le nom et l'histoire des momies qu'il découvre, l'indigène égyptien n'étant bon, semblet-il, qu'à se livrer au trafic mercantile des corps non identifiés qu'il pille ${ }^{7}$. Un égyptologue britannique va même jusqu'à baser la suprématie de la race blanche sur l'étude des momies égyptiennes. Disciple du scientifique américain Samuel Morton, dont le travail porte sur la mesure du volume crânien des différentes races, George Gliddon mesure celui des momies égyptiennes qui, selon lui, présentent elles aussi des caractéristiques raciales différentes. Il conclut que les races étaient aussi inégales au temps de l'Égypte ancienne que dans le Sud des États-Unis contemporains (Pringle, 2001, p. 180-181). Gliddon est également tristement célèbre de l'autre côté de l'Atlantique pour une séance de débandelettage ratée à Boston, au cours de laquelle la princesse égyptienne qu'il avait annoncée s'avère être un homme! (Pringle, 2001, p. 183.) Connaissant son rôle symbolique dans l'entreprise de colonisation, rien d'étonnant à ce que la momie entre en littérature comme figure essentielle de la vengeance et du mal.

\section{PERSONNAGE CENTRAL}

DE L'IMPERIAL GOTHIC

Patrick Brantlinger définit l'Imperial Gothic comme un genre littéraire caractéristique de la fin du $\mathrm{XIX}^{\mathrm{e}}$ siècle qui associe l'intrigue du roman d'aventures 
à des éléments gothiques (Hurley, 2008, p. 196; Brantlinger, 1988, p. 227). Dans son analyse de la momie victorienne, Kelly Hurley étudie les plus importantes de ces mummy fictions et tente de déterminer les différentes problématiques culturelles soulevées par ces créatures. La première, qui renvoie aux origines du mot «fétiche» dans les premiers échanges interculturels en Afrique de l'Ouest au Moyen Âge, réside dans l'impossibilité de traduire la valeur culturelle d'un objet. Pour l'indigène moderne, la momie est un ancêtre, un parent éloigné dont le cadavre est violé par la science occidentale, qui considère quant à elle la momie comme un objet d'étude. Ainsi, dans The Green Mummy de Fergus Hume (1908), le professeur Braddock, égyptologue qui compare les trésors de l'Égypte à ceux du Pérou, se heurte à l'opinion du péruvien Don Pedro qui veut récupérer la momie de son ancêtre, considérée par le professeur comme un spécimen particulièrement intéressant dont l'étude exige le débandelettage (Hurley, 2008, p. 191).

La momie incarne également la problématique de l'antagonisme entre la science et la magie, qui existe depuis l'apparition de la théorie darwinienne et le développement d'une vision matérialiste du réel, dépourvue de dimension spirituelle. Paradoxe vivant, la momie est à la fois un miracle de la science et une preuve de ses limites, mais la littérature résout cette contradiction en la ramenant à la vie, la rendant ainsi maîtresse d'une science autrement plus puissante et dangereuse. C'est le cas de Lyostrah, le terrible chef des myrvoniens dans King of the Dead : A Weird Romance de Frank Aubrey (1903). Le personnage est à la fois scientifique et magicien et utilise les découvertes du XIX $^{\mathrm{e}}$ siècle (notamment concernant l'électricité) pour les développer en vue d'un usage métaphysique, un pouvoir qu'il entend utiliser pour réveiller une armée de momies et envahir l'Angleterre et le monde (Hurley, 2008, p. 180-192).

Enfin, la momie incarne un certain nombre de craintes concernant la chute de l'Empire britannique. Dans une logique de différenciation raciale entre les anciens Égyptiens, considérés comme les ancêtres bibliques des Européens actuels, et les Égyptiens modernes, dont l'infériorité est nécessaire à la justification de l'occupation de leur pays, les momies égyptiennes sont bien souvent blanches dans les romans du gothique impérial. Elles sont ainsi des objets d'identification des doutes des Victoriens quant à la solidité de leur Empire colonial et l'intégrité de leur structure sociale. De ce point de vue, la survie de la momie ou son réveil ne sont que provisoires, et la condition matérielle du cadavre finit toujours par se manifester, à l'image de la fatale disparition de l'Empire. Ainsi, dans She de H. Rider Haggard (1886), deux explorateurs anglais découvrent en Afrique les traces d'une civilisation disparue, dont les descendants actuels, de race noire, sont dominés par la reine blanche Ayesha. Immortelle, elle attend le retour de son amant Kallikrates, prêtre égyptien qu'elle reconnaît en Léo, l'un des deux héros du roman. Mais à l'image des momies qui servent de torches humaines aux danses fétichistes des sauvages actuels (Haggard, 1886, p. 264265), l'illusion de l'éternité ne dure guère, et dans une scène d'horreur suprême, Ayesha, entourée du feu de la Vie censé donner l'immortalité à Léo, s'immole et se ratatine sous le poids insupportable de ses années (Haggard, 1886, p. 355-357). Étrange momie vivante, la reine blanche est paradoxalement réduite à néant par crémation.

\section{SURVIVANCE HOLLYWOODIENNE \\ DU MYTHE}

Ce potentiel spectaculaire de la momie est pleinement exploité par Hollywood, qui fait revivre le monstre du XIX ${ }^{\mathrm{e}}$ siècle. Renan Pollès associe le mythe de la momie à celui de deux autres "morts-vivants" victoriens: le Vampire et Frankenstein (Pollès, 2001, p. 274). Il rappelle que ces trois monstres apparaissent en littérature dans le premier quart du $\mathrm{XIX}^{\mathrm{e}}$ siècle: Polidori écrit The Vampyre en 1819, Mary Shelley publie Frankenstein; or the Modern Prometheus en 1818, et Jane Webb fait paraître The Mummy! A Tale of the Twenty-Second Century... en 1827. Les trois personnages connaissent ensuite une nouvelle vague de succès à la fin $\mathrm{du} \mathrm{XIX}^{\mathrm{e}}$ siècle, avant de devenir des

LA MOMIE INCARNE ÉGALEMENT LA PROBLÉMATIQUE DE L'ANTAGONISME ENTRE LA SCIENCE ET LA MAGIE, QUI EXISTE DEPUIS L'APPARITION DE LA THÉORIE DARWINIENNE ET LE DÉVELOPPEMENT D’UNE VISION MATÉRIALISTE DU RÉEL, DÉPOURVUE DE DIMENSION SPIRITUELLE. mythes populaires dans le premier tiers du $\mathrm{XX}^{\mathrm{e}}$ siècle, grâce au nouveau médium du cinéma. Dans le cas de la momie, ce dernier regain d'intérêt est indissociable de la découverte de la tombe de Toutankhamon en 1922, et de la malédiction supposée qui frappe alors un certain nombre des hommes qui ont été en contact avec le trésor ou la momie du pharaon. Cet événement historique relance l'engouement pour l'Égypte et permet d'établir le roman de momie comme genre littéraire, avant d'en faire un mythe cinématographique (Pollès, 2001, p. 175-189).

On attribue généralement la sortie, par Universal, de Frankenstein de James Whales et de Dracula de Tod Browning en 1931 à la crise de 1929 et à l'ébranlement du capitalisme et du rationalisme scientifique qui s'ensuivent (Pollès, 2001, p. 195). Ces questionnements d'ordre social et idéologique rappellent les préoccupations victoriennes. The Mummy, de Karl Freund, sort en 1932 et participe de cette angoisse sociale. Il raconte la résurrection du grand prêtre Im-Ho-Tep par un archéologue britannique en 1921, la momie ressuscitée cherchant par la suite à ramener à la vie son amour défunt, Anck-es-en-Amon, en tuant pour cela sa réincarnation moderne qui se trouve être une jeune fille anglaise. Dans les années 1950, la Hammer anglaise rachète les droits d'Universal pour faire revivre ses horribles monstres en couleur (Pollès, 2001, p. 216), et aux remakes de Frankenstein et Dracula s'ajoutent celui de The Mummy en 1959. L'histoire se déroule cette fois-ci en 1895 et la momie a davantage le caractère d'un zombie, manipulée par un Égyptien moderne qu'elle finit par éliminer, faisant ainsi preuve d'un certain libre arbitre.

L'une des dernières versions de The Mummy, réalisée par Stephen Sommers en 1999, est également à rattacher à un certain regain d'intérêt pour les mortsvivants victoriens après le Dracula de Francis Ford Coppola (1992) et le Frankenstein de Kenneth Branagh (1994). Le film reprend les éléments essentiels de l'œuvre de Freund et déploie toute la technologie moderne pour donner à voir un véritable film d'aventures, où les scènes d'action spectaculaires se succèdent. L'idée d'amour éternel est toujours présente, avec le désir de la momie Imhotep de ressusciter la défunte Anck-Su Namun, et l'horreur du corps mort revenant à la vie est renforcée par le réalisme des images de synthèse. Pollès remarque que la momie de ce dernier remake se nourrit des chairs de ses victimes pour reconstituer son corps, ce qui est une sorte de «revanche» symbolique sur les mangeurs de momie du Moyen Âge (Pollès, 2001, p. 222-224). 
Le cadavre momifié continue donc de fasciner, au-delà des époques, sans doute par sa capacité à cristalliser une angoisse indissociable de la condition humaine: celle de la mort. Comme le rappelle Pollès, les sociétés industrielles se caractérisent par l'impossibilité de canaliser l'angoisse de la mort et de la cadavérisation (Pollès, 2001, p. 273). La disparition des anciennes traditions laisse la place en Occident à une Église qui reste très vague quant à la réalité du Paradis, par opposition à un Enfer qui, lui, fait l'objet de nombreuses descriptions. La portée de la révolution industrielle en Grande-Bretagne expliquerait donc le succès des momies égyptiennes, que ce soit dans les amphithéâtres lors de séances de débandelettage, ou sur les planches des freak shows. Fétiche impérial, la momie victorienne en vient également à représenter la domination culturelle de l'Empire sur ses sujets égyptiens, la couronne se trouvant finalement menacée par une momie revancharde, symbole d'une puissance occidentale vouée à l'extinction. Cette crainte de la fin d'une domination (néo) impériale sur le monde est peut-être à l'origine d'un retour du film de momie avec les films de Stephen Sommers (The Mummy, 1999, et The Mummy Returns, 2001) puis de Rob Cohen (The Mummy: Tomb of the Dragon Emperor, 2008) ${ }^{8}$. A cette crainte politique s'ajoute l'angoisse de la disparition pure et simple de l'humanité, à la suite des bouleversements climatiques dont elle est l'auteur. La momie incarnerait donc plus que jamais les angoisses d'un nouveau millénaire, et tenterait de figer sur le papier ou la pellicule l'éternité de la Civilisation, faisant alors écho à l'émerveillement de Voltaire, qui affirme à propos des monuments d'Égypte: «Des cadavres ont duré autant que des pyramides » (Voltaire, 1756, introduction, p. XXI).

\section{Bibliographie}

ARNOLD, C. (2006). Necropolis, London and Its Dead, Londres, Simon and Schuster.

AUBREY, F. (1978 [1903]). King of the Dead: A Weird Romance, New York, Arno Press.

BOSSUET, J.-B. (1998 [1689]). Oraisons Funèbres, Paris, Classiques Garnier Multimédia.

BRANAGH, K. (1994). Mary Shelley's Frankenstein, American Zoetrope, 123 min.

BRANTLINGER, P. (1988). Rule of Darkness: British Literature and Imperialism, 1830-1914, Ithaca, NY, Cornell University Press.

BROWNE, J. et S. MESSENGER (2003). "Victorian spectacle: Julia Pastrana, the bearded and hairy female», Endeavour, vol. $27, \mathrm{n}^{\circ} 4$, p. 159.
BROWNING, T. (1931). Dracula, Universal Pictures, $75 \mathrm{~min}$.

BYRON, G.-G. (J.-W. POLIDORI) (1819). «The Vampyre», New Monthly Magazine, avril 1819.

COEN, R. (2008). The Mummy: Tomb of the Dragon Emperor, Universal Pictures, $112 \mathrm{~min}$.

COPPOLA, F.-F. (1992). Bram Stoker's Dracula, American Zoetrope, $128 \mathrm{~min}$.

FREUD, S. (1981 [1920]). «Au-delà du principe de plaisir », Essais de psychanalyse, Paris, Éditions Payot.

FREUND, K. (1932). The Mummy, Universal Pictures, 73 min.

GAUtier, T. (1840). "Le Pied de la momie ", Le musée des familles, septembre 1840.

GAUTIER, T. (1858). Le Roman de la momie, Paris, L. Hachette.

HAGGARD, H.-R. (2007 [1886]). She, A History of Adventure, Londres, Penguin Books.

HITCHCOCK, A. (1960). Psycho, Shamley Productions, 109 min.

HUME, F. (1908). The Green Mummy, Londres, John Long.

HURLEY, K. (2008). "The Victorian Mummy-Fetish, H. Rider Haggard, Frank Aubrey, and the White Mummy", dans M. TROMP (dir.), Victorian Freaks, The Social Context of Freakery in Britain, Columbus, Ohio University Press, p. 180-199.

MCCLINTOCK, A. (1995). Imperial Leather: Race, Gender, and Sexuality in the Colonial Contest, Londres et New York, Routledge.

POE, E.-A. (1845). «Some Words with a Mummy ", American (Whig) Review, avril.

POLLÈS, R. (2001). La Momie de Khéops à Hollywood, Généalogie d'un mythe, Paris, Les éditions de l'Amateur.

PRINGLE, H. (2001). The Mummy Congress, Science, Obsession, and the Everlasting Dead, Londres, Fourth Estate.

SALAM, A. (1969). Al Mumia (The Mummy), Organisme du cinéma égyptien, $100 \mathrm{~min}$.

SHOHAT, E. et R. STAM (2007 [1994]). Unthinking Eurocentricism, Multiculturalism and the Media, New York et Oxon, Routledge.

SOMMERS, S. (1999). The Mummy, Universal Pictures, 125 min.

SOMMERS, S. (2001). The Mummy Returns, Universal Pictures, $130 \mathrm{~min}$.

STERN, R. (2008). "Our Bear Women, Ourselves, Affiliating with Julia Pastrana» dans M. TROMP (dir.), Victorian Freaks, The Social Context of Freakery in Britain, Columbus, Ohio University Press, p. 200233.

THOMAS, L.-V. (1985). Rites de mort, Pour la paix des vivants, Paris, Fayard.
VOLTAIRE (1756). "Introduction, XXI, Des monuments des Égyptiens ", Essai sur les mours et l'esprit des nations, et sur les principaux faits de l'histoire depuis Charlemagne jusqu'à Louis XIII.

WEBB, J. (1827). The Mummy! A Tale of the Twenty-Second Century..., Londres, Henry Colburn.

WHALE, J. (1931). Frankenstein, Universal Pictures, $71 \mathrm{~min}$.

\section{Notes}

1. Renan Pollès parle de la «La Momie des Apothicaires» (Pollès, 2001, p. 13-32). Son ouvrage La Momie de Khéops à Hollywood, Généalogie d'un mythe fut d'une utilité précieuse pour la rédaction de cet article.

2. Il s'agit pour Pollès de la «La Momie des Antiquaires» (Pollès, 2001, p. 33-80).

3. Freud définit le principe de plaisir comme la tendance pour l'appareil psychique à «maintenir basse la quantité d'excitation, tout ce qui est propre à accroître celle-ci [étant] nécessairement ressenti comme opposé à la fonction de l'appareil, c'est-à-dire comme déplaisant » (Freud, 1920, 45).

4. Cette attitude va donc à l'encontre du fonctionnement «normal» de l'appareil psychique que Freud décrit comme suit: "Sous l'influence de l'instinct de conservation du moi, le principe de plaisir est relayé par le principe de réalité; celui-ci ne renonce pas à l'intention de gagner finalement du plaisir mais il exige et met en vigueur l'ajournement de la satisfaction, le renoncement à toutes sortes de possibilités d'y parvenir et la tolérance provisoire du déplaisir sur le long chemin détourné qui mène au plaisir » (Freud, 1920, p. 46).

5. Cette loi permet d'augmenter considérablement le nombre de cadavres mis légalement à disposition des facultés de médecine. Avant cela, le Murder Act de 1752 autorise uniquement la dissection des corps de criminels exécutés par la justice.

6. La littérature française exploite cette dimension avec les deux récits fondateurs de Théophile Gautier: Le Pied de la momie (1840) et Le Roman de la momie (1858), qui jouent sur le potentiel du corps momifié à mêler désirs érotique et morbide (Pollès, 2001, p. 118-119).

7. Le film Al Mumia (The Mummy), réalisé en 1969 par Abdel Salam, offre un contre-point postcolonial nécessaire à l'appréhension historique des fouilles archéologiques. Le réalisateur s'attache en effet à montrer la situation complexe des Égyptiens au XIX ${ }^{\mathrm{e}}$ siècle, partagés entre la nécessité économique de faire commerce des momies, et le respect pour leurs ancêtres (Shohat et al., 2007, p. 153-156).

8. De ce point de vue, le fait que le film de Rob Cohen ait pour cadre la Chine et pour intrigue, la résurrection d'un empereur chinois n'est pas anodin. 